\title{
PERFIL EMPRENDEDOR Y PRECARIO DE DESEMPLEADOS QUE SE AUTOEMPLEAN
}

Octavio Martín Maza Cortés*, Laura Cecilia Gil Paredes**

* Profesor Investigador del Departamento de Sociología y Antropología. Universidad Autónoma de Aguascalientes.

${ }^{* *}$ Research Student at the Department of Sociology and Anthropology. University of Haifa.

Dirección para recibir correspondencia: octaviomazadc@gmail.com 


\section{RESUMEN}

Objetivo: Identificar si los desempleados que se autoemplean poseen un perfil emprendedor conjuntamente con su perfil precario.

Material y método: Se realizó un análisis cualitativo de contenido de 30 relatos escritos de personas desempleadas. Debido a su carácter cualitativo, la muestra no tuvo como objeto la generalización probabilística de los resultados, sino su transferencia a otros contextos. El estudio compara desempleados que se autoemplearon con los que consiguieron un empleo. Para determinar el perfil precario, se analizó el significado que los sujetos atribuyen al desempleo tanto en el aspecto económico como en el subjetivo. Los factores para determinar el perfil emprendedor incluyeron género, edad, estado civil, nivel socioeconómico, nivel educativo, capital social y, sobre todo, locus interno de control que es un rasgo de emprendedurismo.

Resultados: Con respecto al perfil precario, en el aspecto económico, el desempleo significó pobreza, en distintos grados y subjetivamente, inseguridad y vulnerabilidad. En ambos sentidos, los desempleados más precarios fueron los jefes de familia sin otro ingreso en el hogar, los mayores de 40 años o quienes sólo poseen escolaridad básica. Fueron ellos quienes en su gran mayoría optaron por el autoempleo. En cuanto al perfil emprendedor, el análisis reveló que la mayoría de los autoempleados y de quienes obtuvieron empleo presentaron locus interno de control.

Conclusiones: De acuerdo con los resultados, el autoempleo se revela más como una opción precaria que emprendedora. Parece que el impulso hacia el autoempleo es la precariedad; sin embargo, también se puede sostener que los desempleados más precarios superan el desempleo gracias al rasgo emprendedor del locus interno de control. Por ello, resulta relevante reconocer no sólo el perfil precario, sino también el perfil emprendedor de quienes se han visto forzados a autoemplearse, para apoyarlos de acuerdo con sus necesidades específicas y su talento empresarial.

Palabras clave: Emprendedurismo. Desempleo. Autoempleo. Perfil. Empresa. 


\section{ABSTRACT}

Objective: To ascertain if unemployed people who become self-employed have an entrepreneurial profile jointly with their precarious profile.

Material and method: A qualitative content analysis of 30 written stories of unemployed persons was carried out. Due to its qualitative character, the objective of the sample was not to generalize the results probabilistically, but to transfer them to other contexts. The study compares unemployed people who became self-employed to unemployed people who found a job. To determine the precarious profile of the individual, the analysis focused on what unemployment meant economically and subjectively to each one. The factors for determining the entrepreneurial profile included gender, age, marital and socioeconomic status, education, social capital differences, and most importantly, internal locus of control, which is an entrepreneurial trait.

Results: In regards to the precarious profile, unemployment meant various degrees of poverty economically, and subjectively, it meant insecurity and vulnerability. In both senses, the most precarious unemployed people were the heads of families with no other household source of income, those over 40 years of age, or those who had only received an elementary education. The majority of these individuals went on to become self-employed. Concerning the entrepreneurial profile, the analysis found that the majority of both the self-employed and those who found a job possessed internal locus of control.

Conclusions: The results show self-employment to be a more precarious than an entrepreneurial option. It is precariousness that seems to drive individuals to self-employment. However, it could also be said that the most precarious unemployed people overcame unemployment because they possessed internal locus of control, an entrepreneurial trait. From the above, it is important to recognize not only the precarious profile of those who have been forced into self-employment, but also their entrepreneurial profile, in order to support them according to their specific needs and entrepreneurial talent.

Keys Word: Entrepreneurial. Unemployment. Self-employed. Profile. Enterprises. 


\section{Introducción}

La relación entre el desempleo y el autoempleo ha sido estudiada por sociólogos y economistas principalmente desde una perspectiva macroeconómica. Desde dicho enfoque, algunos estudios asocian el desempleo con mayores tasas de autoempleo y apertura de negocios (Parker, 2009; Storey and Jones, 1987). Sin embargo, otros autores sostienen lo contrario, con base en la opinión de que los desempleados tienden a ser individuos sin el capital humano y el talento empresarial necesario para crear un negocio (Audretsch et al., 2001).

Una de las explicaciones macroeconómicas a la relación entre autoempleo y desempleo proviene del Global Entrepreneurship Monitor (GEM), que distingue entre emprendedor por necesidad y por oportunidad. El emprendedurismo por necesidad, que sería propiamente el autoempleo, consiste en "tener que convertirse en empresario (o autoempleado) porque "no se tiene una mejor opción"'” (Acs, 2006, p. 97). El emprendedurismo por oportunidad es "una elección activa de iniciar una nueva empresa, con base en la percepción de que existe una oportunidad no explotada 0 subexplotada" (ibid).

El GEM ha encontrado que cuando se elevan las tasas de desempleo y otras alternativas de empleo son escasas, habría también un incremento de emprendedurismo por necesidad. De acuerdo con el GEM esta clase de emprendedurismo no tiene efectos en el desarrollo económico, mientras el emprendedurismo por oportunidad tiene un positivo y significativo efecto (ibid); es decir, mientras menos desarrollado es un país, posee mayor número de emprendedores por necesidad (autoempleados) (Acs, 2006, p. 102). Aunado a lo anterior, estudios realizados en México señalan que mientras más pobres son dichos autoempleados, más tienden a trabajar en la informalidad, que es el sector en condiciones más precarias de trabajo, fundamentalmente por falta de seguridad social (Rodríguez-Oreggia, 2007, p. 42).

Sin embargo, en México, también se ha confirmado que, aunque el autoempleo no genere desarrollo económico (debido a que es "por necesidad"), éste actúa como un refugio ante la falta de empleo. Es decir, incluso considerando que cerca del 12\% de los desempleados se autoemplean en microempresas del sector informal (Arceo, 2011, pp. 34-35), que se caracteriza por su escasa innovación y baja productividad (Lederman et al., 2014; Salas, 2003), dichos 
emprendimientos generan el 40\% del empleo en México, según la Organización para la Cooperación y el Desarrollo Económico (OCDE, 2013).

Con respecto a cómo influyen las características de los individuos en la relación del desempleo con el autoempleo, la literatura muestra que el perfil del autoempleado, además de características demográficas específicas como ser hombre, casado y mayor de cuarenta años (Dunn and HoltzEakin, 2000; Arum and Müller, 2009; Parker, 2009); comprende determinadas aptitudes, provenientes de su capital humano (contar con educación superior) (Baycan-Levent et al., 2006; Blanchflower, 2004), y capital social (recurso a redes sociales de apoyo) (Davidsson and Honig, 2003; Parker, 2009). A lo anterior, se suman rasgos de personalidad como la tolerancia al riesgo (Douglas and Shepherd, 2002; Parker, 2009), y el locus interno de control. El locus de control es un concepto acuñado por Rotter (1966), y se ha convertido en uno de los rasgos de personalidad más estudiados en cuanto a su influencia en el inicio de un negocio. El locus interno de control "se refiere al grado en que las personas creen que (...) el resultado de su comportamiento depende de su propio comportamiento o características personales" (Rotter, 1966, cit. en: Rotter, 1990, p. 489). El locus externo de control "se refiere al grado en el cual las personas creen que (...) el resultado es una función de la casualidad, la suerte o el destino, está bajo el control o el poder de otros, o es simplemente impredecible" (ibid).

Las características antes mencionadas varían dependiendo del país. En el caso de México, el reporte del GEM 2013, señala que el perfil emprendedor es el de hombres o mujeres, con tendencia a ser cada vez más jóvenes y educados y que emprenden por oportunidad (Naranjo et al., 2013, p. 63). En general, en México se asocia locus interno de control con intención emprendedora entre estudiantes universitarios (Correa y Aduna, 2012; Espíritu, 2011). Sin embargo, también se ha encontrado que las características psicológicas que distinguen a los autoempleados de los asalariados son la orientación al logro y la tolerancia al riesgo, más significativamente que el locus interno de control (Brassiolo y Arreaza, 2013, p. 87). No obstante, mientras el locus interno de control es un rasgo indiscutiblemente emprendedor, no se puede afirmar lo mismo con respecto a la aversión al riesgo ${ }^{1}$ (Caliendo, Fossen y Kritikos, 2014).

\footnotetext{
${ }^{1}$ De ahí que se viera más conveniente no incluir la aversión al riesgo en un estudio como el presente, pues se estimó que iría en detrimento de la claridad de los resultados.
} 
Con frecuencia, la falta de impacto económico del emprendedurismo por necesidad se relaciona con la baja calidad de los micronegocios que genera (Storey and Johnson, 1987). Como se acaba de explicar, esto se ha observado en México, donde se crean una gran cantidad de micronegocios informales. Al mismo tiempo, la baja calidad del emprendedurismo por necesidad se asocia con las escasas aptitudes empresariales de los individuos (Block and Sandner 2009, cit. en: Stephan, Hart and Drews, 2015). Así, se llega a sostener que los emprendedores por necesidad no serían propiamente emprendedores (Sanguinetti, 2013, pp. 38-39; Acs, 2006).

No obstante, también es verdad que no todos los desempleados, aun teniendo necesidad de trabajo, se convierten en autoempleados. En este artículo, se asume que ello puede deberse a que quienes se autoemplean cuentan con un perfil emprendedor específico. Con base en la teoría del trabajo precario se sostiene que, los emprendedores por necesidad se han visto forzados a autoemplearse, y por tanto, se encuentran en una situación de precariedad, tal condición no significa que sean individuos sin rasgos emprendedores; es decir, que los autoempleados son precarios, pero también son emprendedores. Así mismo, presenta los resultados de un estudio cuyo objetivo fue identificar si los desempleados que se autoemplean poseen un perfil emprendedor conjuntamente con su perfil precario.

Con el fin de alcanzar dicho objetivo se realizó un análisis de relatos escritos de personas desempleadas. El análisis incluye una comparación entre los desempleados que se convierten en emprendedores por necesidad y los que consiguen un empleo. En primer lugar, se describe el perfil precario de los desempleados, para después mostrar cómo ante la misma circunstancia adversa (el desempleo), se revela el perfil emprendedor de algunos, que serían los que cuentan con ciertos recursos personales para superar el desempleo por la vía del autoempleo. Principalmente se buscó la presencia de locus interno de control que es uno de los rasgos de personalidad más asociados con el emprendedurismo.

Con este estudio, se espera contribuir al conocimiento del emprendedurismo por necesidad como un comportamiento empresarial. Se asume que, aunque sean distintos a los emprendedores por oportunidad, los autoempleados poseen potencial para contribuir al desarrollo del país si se brindan los apoyos adecuados a su situación. 
En la siguiente sección se presentan algunos datos sobre la situación del desempleo en México. Después se expone la metodología, los resultados del estudio, la discusión y las conclusiones.

\section{El Desempleo en México}

De acuerdo al panorama laboral de los últimos años en México, el autoempleo es una estrategia que los sujetos han puesto en marcha ante la problemática del desempleo. De ahí que, pese a las condiciones del país, la tasa de desocupación en México se mantenga, según datos de la Organización Internacional del Trabajo, como una de las más bajas de América Latina (OIT, 2013), e incluso de los países de la OCDE, los cuales alcanzan en promedio un 9\% (OCDE, 2014); salvo los incrementos que se observaron en julio y septiembre de 2009, las tasas de desempleo en México se han mantenido con una tendencia constante a la baja: el promedio para 2009 fue de 5.4\%, en 2010 de 5.3\% y para 2011 de tan sólo 5.2\%. En este período, de acuerdo a los datos del Instituto Nacional de Geografía y Estadística, desagregados por sexo, se observa que, para los hombres, pasó de 4.8\% a 4.6\%, y para las mujeres, de 5.2\% a 4.3\% (INEGI, 2012).

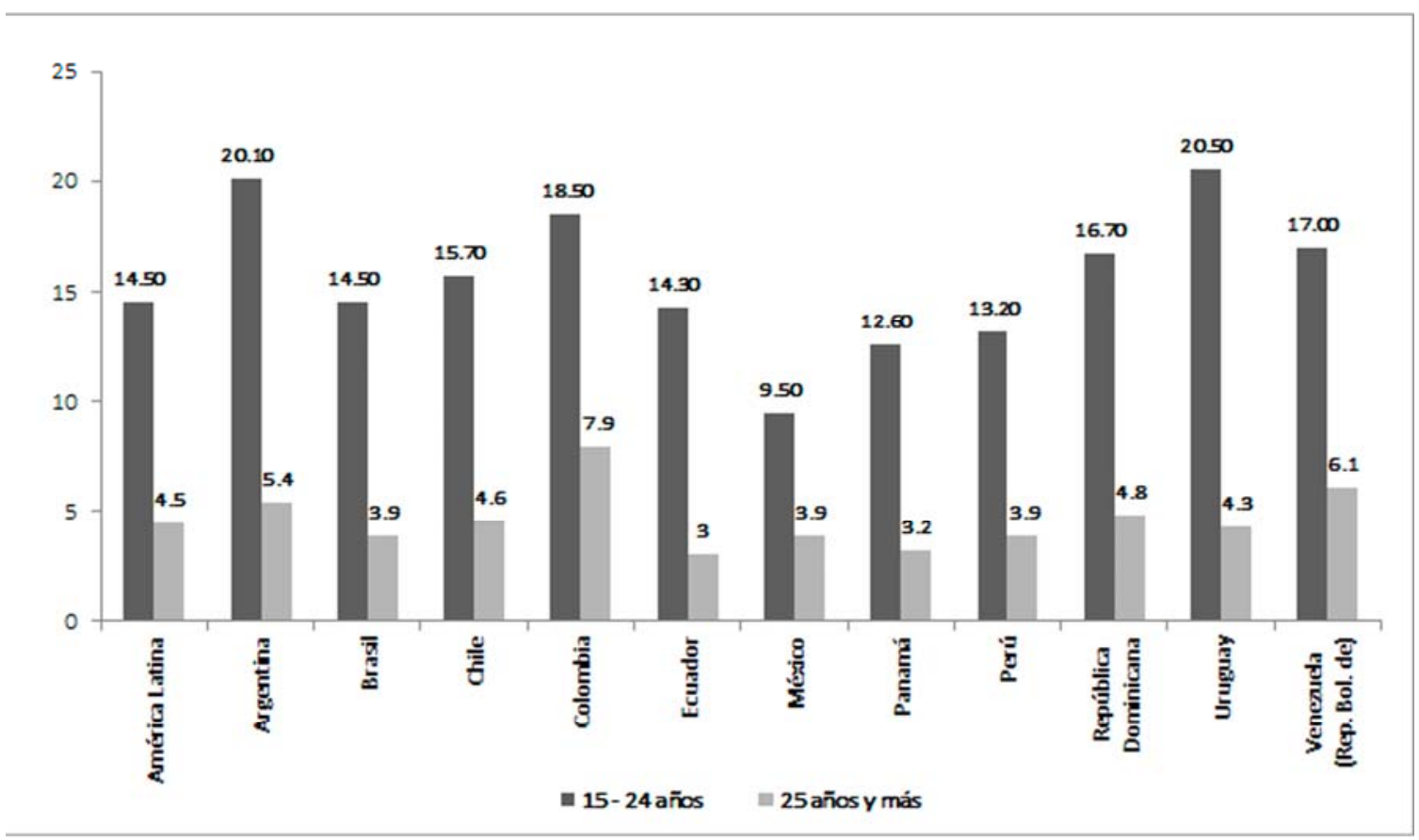

Figura 1: América Latina: tasa de desempleo urbano por grupo etario, enero-septiembre 2012-2013. Fuente: Tomado de "Panorama Laboral 2013. América Latina y el Caribe", OIT. 


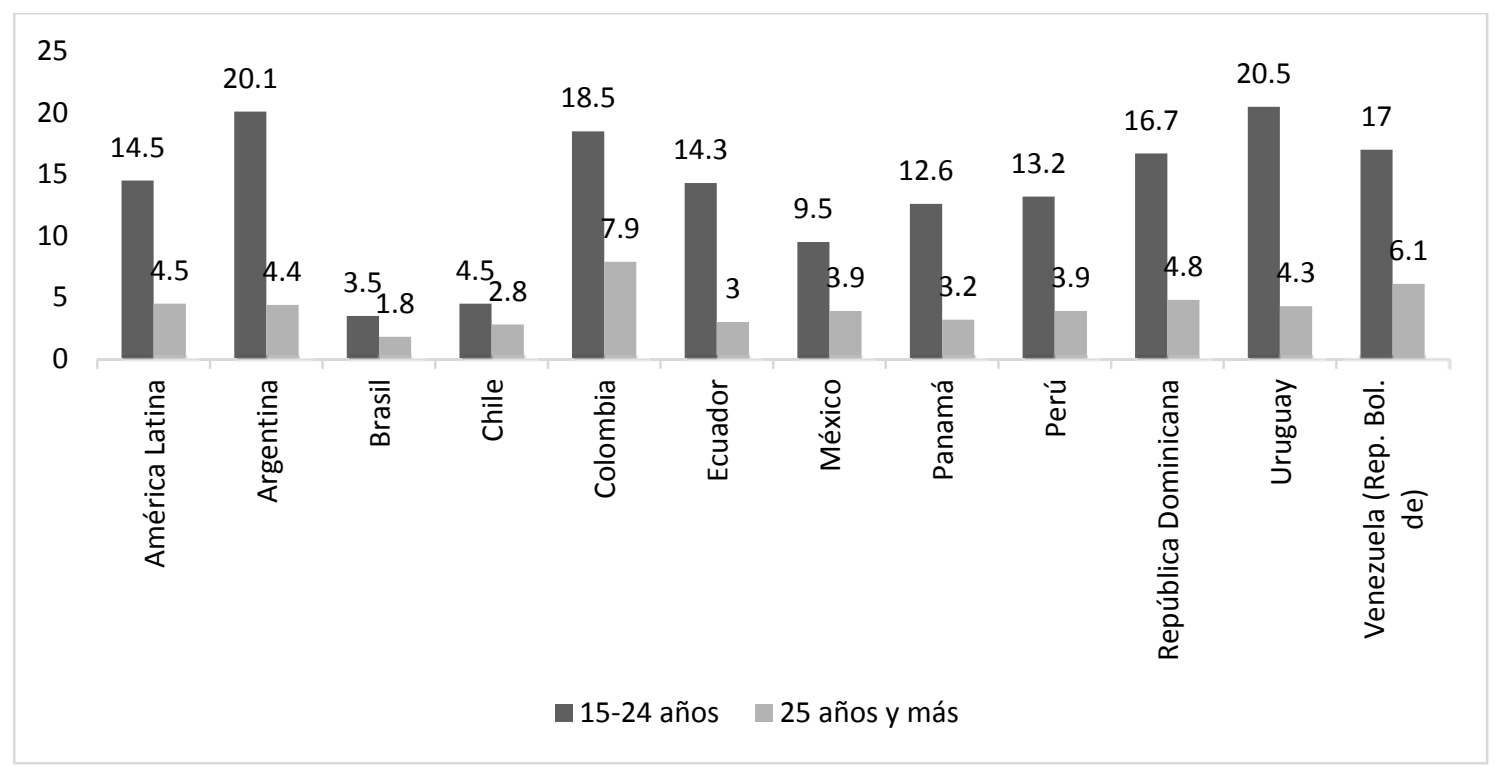

Figura 1: América Latina: tasa de desempleo urbano por grupo etario, enero-septiembre 2012-2013. Fuente: Tomado de "Panorama Laboral 2013. América Latina y el Caribe", OIT.

Sin embargo, si se atiende a los datos por grupo de edad, se observa que, a pesar de haberse reducido un $2.2 \%$, la tasa más alta se concentra entre los jóvenes: en septiembre de 2009 fue de $12.31 \%$ y a finales de año quedó en $9.24 \%$. Un año después, en noviembre de 2010 , la tasa de desocupación fue de 5.62\% respecto a la Población Económicamente Activa (PEA), (INEGI, 2012) (ver tabla 1). De acuerdo con la OIT (2013), son los jóvenes los que continúan integrando el grupo etario con mayores problemas de empleo, tanto por sus niveles altos de desempleo, como por la permanencia de altos porcentajes en el empleo informal. Además, se señala que un período de desempleo prolongado, para los jóvenes, puede conducir al desaliento con los siguientes efectos negativos en sus competencias y experiencia laboral. Para el caso de México, la tasa de desempleo del grupo de 15 a 24 años es de 9.5\% a comparación de la del grupo de 25 años y más que es de un 3.9\% (ver figura 1).

Tabla 1

Tasas de desocupación en México, 2009-2010 


\begin{tabular}{|c|c|c|c|c|c|c|c|c|}
\hline \multirow[b]{3}{*}{ Grupos de edad } & \multicolumn{8}{|c|}{ Tasas de desocupación trimestral en México por grupos de edad, 2009 - 2010} \\
\hline & \multicolumn{4}{|c|}{2009} & \multicolumn{4}{|c|}{2010} \\
\hline & I & II & III & IV & I & II & III & IV \\
\hline Total & 5.06 & 5.17 & 6.24 & 5.33 & 5.33 & 5.27 & 5.62 & 5.36 \\
\hline 14 a 19 años & 9.98 & 9.92 & 12.31 & 9.24 & 9.48 & 10.121 & 11.29 & 10.46 \\
\hline 20 a 29 años & 7.82 & 7.95 & 9.5 & 8.23 & 8.25 & 7.91 & 8.76 & 8 \\
\hline 20 a 24 años & 9.03 & 9.77 & 11.13 & 9.57 & 9.48 & 8.92 & 10.36 & 8.93 \\
\hline 25 a 29 años & 6.66 & 6.21 & 7.95 & 6.91 & 7.06 & 6.92 & 7.15 & 7.08 \\
\hline 30 a 39 años & 4.22 & 4.23 & 5.17 & 4.51 & 4.71 & 4.45 & 4.53 & 4.51 \\
\hline 30 a 34 años & 4.58 & 4.77 & 5.52 & 4.94 & 5.19 & 4.91 & 5.1 & 4.66 \\
\hline 35 a 39 años & 3.87 & 3.71 & 4.83 & 4.09 & 4.24 & 4.01 & 3.98 & 4.37 \\
\hline 40 a 49 años & 3.26 & 3.49 & 4.09 & 3.77 & 3.6 & 3.67 & 3.3 & 3.37 \\
\hline 40 a 44 años & 3.52 & 3.51 & 4.18 & 3.81 & 3.77 & 3.69 & 3.47 & 3.19 \\
\hline 45 a 49 años & 2.95 & 3.47 & 3.99 & 3.71 & 3.4 & 3.64 & 3.09 & 3.58 \\
\hline 50 a 59 años & 3.13 & 3.07 & 3.8 & 3.14 & 3.04 & 3.18 & 3.5 & 3.54 \\
\hline 50 a 54 años & 3.12 & 3.16 & 3.95 & 3.21 & 2.95 & 2.93 & 3.56 & 3.68 \\
\hline 55 a 59 años & 3.14 & 2.94 & 3.58 & 3.03 & 3.18 & 3.54 & 3.41 & 3.34 \\
\hline 60 años y más & 1.72 & 2.33 & 2.24 & 1.95 & 1.97 & 1.98 & 2.21 & 2.55 \\
\hline 60 a 64 años & 2.33 & 3.35 & 2.59 & 2.73 & 2.5 & 2.61 & 3.1 & 3.25 \\
\hline 65 años y más & 1.25 & 1.56 & 1.98 & 1.34 & 1.55 & 1.48 & 1.52 & 1.99 \\
\hline No especificado & & 0.55 & 2.07 & 3.76 & 3.33 & 6.15 & 9.67 & 2.44 \\
\hline
\end{tabular}

Fuente: INEGI, Encuesta Nacional de Ocupación y Empleo.

En lo que refiere a la condición de actividad para el año 2010, la subocupación disminuyó a 8.5\% respecto a la población ocupada, lo que representa una reducción de 1.13 puntos porcentuales, comparado con junio de 2009. En noviembre de ese año la subocupación descendió a 7.1\% (INEGI, 2010). Lo anterior, quiere decir que se registró un mayor porcentaje de personas que encontraron un trabajo de tiempo completo en relación con los meses pasados. De acuerdo con la información estadística presentada, se podría sostener que, comparado con otros países, el desempleo en México no es un problema grave, debido a que corresponde a una pequeña proporción respecto a la población económicamente activa. Sin embargo, la perspectiva de esta estimación cambia si se realiza un análisis sobre los tipos de empleos a los que recurren los individuos para no permanecer en la desocupación por periodos prolongados.

De acuerdo a la Secretaría del Trabajo y Previsión Social (STPS), durante el segundo trimestre de 2014, los datos de los ocupados por posición en el trabajo fueron los siguientes: los que trabajaron por cuenta propia representaron el 22\%, otro $4 \%$ fueron empleadores, el $68 \%$ estuvieron empleados como asalariados y el 6\% no recibieron ingresos (STPS, 2014) (ver figura 
2). Aunque es verdad que según estos datos la mayoría se ocupa como asalariado, existen otras evidencias que muestran que "casi el $70 \%$ de quienes regresan a un empleo, lo hacen en unidades donde laboran no más de 5 personas" (Salas, 2003, p. 139). De modo que los micronegocios constituyen una verdadera alternativa de empleo para un gran porcentaje de trabajadores en el país. Sin embargo, son ésos los espacios con las peores condiciones laborales. De acuerdo a información de la Encuesta Nacional de Ocupación y Empleo (ENOE), es en el sector de los hogares donde se albergan la mayoría de los micronegocios y donde se concentran las actividades informales. En términos generales, puede concluirse que en México el 34.6\% de la población ocupada se concentra en el sector de los hogares, de los cuales el $76.7 \%$ se encuentra en la informalidad² (Arceo, 2011).

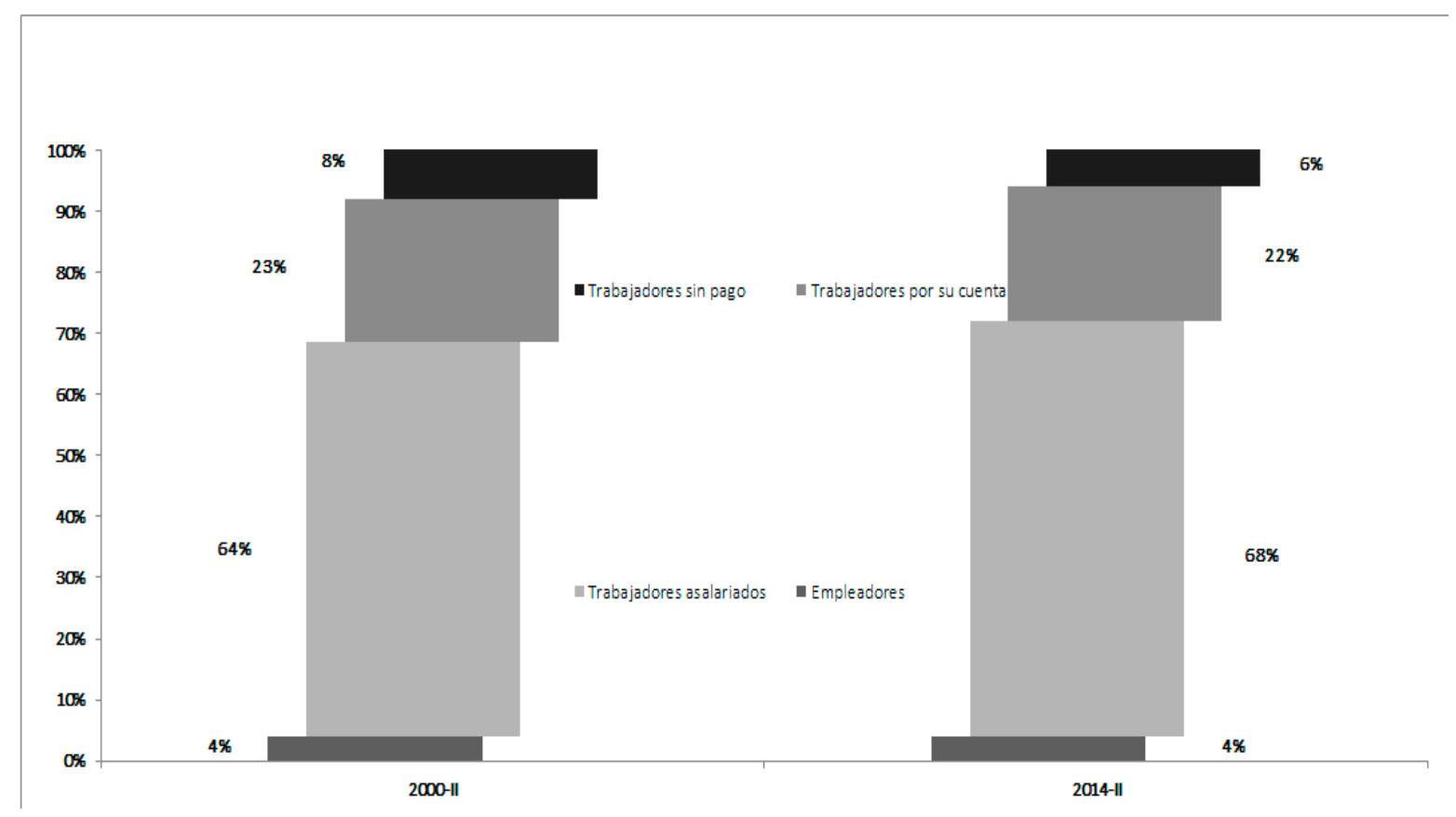

Figura 2. Población ocupada por posición en el trabajo.

Fuente: Tomado de "Información laboral 2014", STPS, 2014.

Las empresas en el sector informal se caracterizan por su baja productividad, pues "carecen de acceso a créditos y buscan no ser visibles en vez de crecer" (OCDE, 2013, p. 15). Sin embargo,

2 "Los individuos informales se definen como aquellos sin acceso a instituciones de salud públicas" (Arceo, 2011, 34). 
a pesar de su baja productividad, las microempresas generan el 40\% del empleo en México (Ibid, p. 14). Por otro lado, según la ENOE, los desempleados que transitan al autoempleo informal aumentan ligeramente en períodos de recesión (2008-2010), con respecto a períodos de expansión (2005-2007): el porcentaje de hombres pasó de $11.5 \%$ a 11.64\% y el de mujeres, de $5.29 \%$ a 5.89\% (Arceo, 2011, pp. 34-35).

Por lo tanto, en México, el autoempleo no es creador de empleos de calidad, sino ahorrador de desempleo. En este artículo, se sostiene que esto es así porque los autoempleados son precarios; sin embargo, también se asume que cuentan con capacidad emprendedora.

A continuación, se presenta un breve apartado sobre la teoría del Trabajo Precario que da soporte a las afirmaciones anteriores.

\section{El Trabajo Precario}

La Teoría del Trabajo Precario proviene de la Teoría de la Regulación. Nacida en Francia en los años setenta, esta teoría trata de explicar, basada en el concepto de regulación, cómo son posibles los períodos prolongados de estabilidad en el capitalismo, a pesar de sus contradicciones y crisis (De la Garza, 2001). Dicha teoría plantea la transición de un período dominante de desarrollo capitalista en crisis, a un "nuevo" paradigma productivo; es decir, los nuevos regímenes de acumulación y formas de organizar el trabajo emergerían para superar la crisis $^{3}$ (Amín y Robins, 1991).

Los regulacionistas encuentran dos causas de la crisis presentada por el capitalismo en los años 80's: la primera sería la crisis de los procesos de producción provocada por la rigidez del gigantismo, que trae consigo una elevación de los costos y del trabajo improductivo. La segunda sería la crisis de la regulación entre producción y consumo; es decir, debido a la rigidez salarial, se elevaron los costos de producción sin que se diera un incremento equivalente en la productividad, con lo cual cayó la tasa de ganancia, provocando una crisis de rentabilidad (De la Garza, 2001).

\footnotetext{
3 El modo de regulación es un conjunto de normas, instituciones, formas de organización, redes sociales y patrones de conducta que contribuyen a la permanencia de un régimen de acumulación. El régimen de acumulación es un patrón de producción y consumo que se reproduce en un período largo de tiempo (De la Garza, 2001).
} 
En este contexto lo que hace posible que las empresas sean rentables es la implementación de trabajo flexible y autónomo, por ser más barato; sin embargo, este tipo de trabajo es precario para el individuo (lbid).

El trabajo precario es aquel trabajo mal remunerado, con duración indefinida (alargada) de la jornada laboral y sin seguro social. Además, en el trabajo precario el único responsable de esos beneficios es el mismo individuo (De Oliveira, 2006; OIT 2012; Frade et al., 2004; Rodgers y Rodgers, 1989); es decir, los sujetos dejan de contar con la seguridad que anteriormente se obtenía a través de un puesto de trabajo (Castel, 1997).

Los teóricos de la precariedad sostienen que la tendencia a la precariedad se generaliza entre todos los grupos poblacionales y, en general, entre todos los tipos y niveles de trabajo, desde los asalariados en el sector formal hasta los temporales y el autoempleo (Kalleberg, 2009; De Oliveira y García, 1998). Sin embargo, no todos los individuos enfrentan la precariedad del mismo modo. Algunos son más vulnerables que otros. Los estudios sobre precariedad laboral destacan características sociodemográficas, como: edad, género, nivel de escolaridad, estado conyugal, que hacen más vulnerables a ciertos sujetos a ocupar los puestos de trabajo menos favorables, mientras que a otros los vuelven más "empleables". Otro factor que se considera fundamental son las redes sociales, con lo cual queda claro que tener trabajo no depende de un juego de la libre oferta y demanda (De la Garza y Gaspar, 2010).

En esta línea, en un estudio sobre precariedad laboral juvenil en México, De Oliveira (2006), ha encontrado que el perfil del trabajador precario sería el de los jóvenes de zonas rurales, con escolaridad básica y procedencia familiar de clase baja.

El autoempleo también es considerado un trabajo precario -de mala calidad- en aspectos como los bajos ingresos, los horarios flexibles (largas jornadas) y la falta de seguridad social. De acuerdo con Beck (2007), esto es así porque el autoempleo asocia la oportunidad con el riesgo; es decir, por un lado, el autoempleo abre nuevas posibilidades de elegir el "propio trabajo" y la propia vida, pero por otro, provoca que los riesgos que en otros tiempos eran asumidos por el Estado y por las grandes empresas ahora deben ser tomados por el individuo (Beck, 2007, p. 80). 
Las implicaciones que ello tiene para los autoempleados, son el aumento de la incertidumbre y el riesgo laborales (Kalleberg, 2009, p. 2).

Por tanto, el autoempleo se considera precario porque el hecho de que el sujeto sea el único responsable de su seguridad laboral implica, por un lado, inseguridad económica y por otro (subjetivamente), vulnerabilidad; estos dos aspectos de la precariedad son los que han sido tomados en cuenta en este estudio, cuya metodología se presenta a continuación.

\section{MATERIAL Y MÉTODO}

Consistió en 43 relatos escritos de personas desempleadas, que se obtuvieron mediante la convocatoria abierta a un concurso; de los 43 fueron excluidos 13, pertenecientes a personas que no encontraron empleo ni se autoemplearon. Aquí se presentan los resultados del análisis cualitativo de los 30 relatos válidos de los 43 recabados. De esos 30 relatos válidos, 16 son de personas que se autoemplearon y 14 de individuos que encontraron un trabajo como empleados.

El método utilizado fue el análisis cualitativo de contenido, de ahí que la muestra no tuviera como objeto la generalización probabilística de los resultados, sino su transferencia a otros contextos. El análisis cualitativo aporta elementos que otras investigaciones difícilmente captan, pues refleja la transición del desempleo al autoempleo desde la experiencia de los desempleados, con base en la identificación de los patrones que la caracterizan (White and Marsh, 2006, p. 15).

Tal como se expuso anteriormente, la precariedad laboral se refiere tanto al aspecto económico como al subjetivo. Por ello, para obtener el perfil precario de los desempleados se analizó el significado que ellos mismos atribuyen a su condición de desempleo en ambos sentidos. Concretamente, en el aspecto económico el desempleo significa pobreza, en distintos grados, y a nivel subjetivo, experimentar sentimientos de inseguridad y vulnerabilidad.

Para obtener el perfil emprendedor de los desempleados que se autoemplearon, se buscó la presencia de locus interno de control que es uno de los rasgos de personalidad más asociados con el emprendedurismo. Después se hizo una comparación con los desempleados que 
consiguieron un empleo, también en lo que respecta a su género, edad, estado civil, nivel socioeconómico, nivel educativo y capital social ${ }^{4}$.

Las categorías de análisis que se utilizaron se presentan en la siguiente tabla:

\section{Tabla 2}

Categorías de análisis. Relatos de desempleados

\begin{tabular}{lll}
\hline Categoría & Subcategoría & Codificación \\
\hline Precariedad & Pobreza & $\begin{array}{l}\text { Reducción de ingresos } \\
\text { familiares } \\
\text { Precariedad económica }\end{array}$ \\
& $\begin{array}{l}\text { Precariedad } \\
\text { subjetiva }\end{array}$ & Sentimientos de inseguridad \\
& & $\begin{array}{l}\text { de vulnerabilidad } \\
\text { Otros sentimientos }\end{array}$ \\
Locus de control & Culpa a: él mismo / otros \\
interno / externo & Dónde está o cuál es la \\
& solución: él mismo / otros \\
\hline
\end{tabular}

4 Davidsson y Honig (2003) definen el capital social como la "habilidad de los actores de extraer beneficios de sus estructuras sociales, redes y relaciones" (Davidsson and Honig, 2003, p. 307). 
Fuente: Elaboración propia, con base en White and Marsh, 2006.

Para detectar el tipo de locus de control se analizó en cada relato la presencia de frases que indicaran si los individuos culpaban a otros o a ellos mismos por su situación de desempleo (categoría "culpa a:"). Se clasificaron como poseedores de locus externo de control a quienes culpaban al gobierno, la situación económica del país, o señalaban que "la vida es así" (fatalismo). La clasificación de locus interno de control fue para quienes mencionaron que el desempleo se debía a su falta de aptitudes, o cualquier otra característica individual (educación, edad).

Para reforzar la asignación de la categoría "interno" o "externo" se cotejó el "culpa a:" con "dónde está o cuál es la solución". Se clasificaron como poseedores de locus externo de control a quienes no sabían cuál era la solución o expresaron que ésta era ajena a ellos mismos: que el gobierno les diera empleo, o que la vida no les hubiera tratado tan mal. Entraron en la clasificación de locus interno de control quienes señalaron que salir del desempleo depende de ellos mismos (de su esfuerzo, trabajo duro, fortaleza en la adversidad).

Las características sociodemográficas de los autores de los relatos fueron: género (femenino, masculino); edad (20 a 30 años; 31 a 40; 41 a 50 o más); estado civil (soltero, casado, divorciado); nivel socioeconómico (el declarado: alto, medio o bajo) y nivel educativo (último grado de estudios: postgrado, profesional, preparatoria, secundaria, primaria).

Por último, como poseedores de capital social fueron clasificados quienes contaron con el recurso a la ayuda familiar o de amigos para superar el desempleo, y sin capital social a quienes lo consiguieron solos.

La codificación de la información fue debidamente validada por los dos autores. La interpretación de los resultados se apoyó en la comparación entre éstos y los hallazgos de otros autores, la cual se muestra en el apartado de discusión.

\section{RESULTADOS}

Perfil precario de los desempleados. 


\section{El desempleo como pobreza económica.}

En los relatos analizados se encontró que el hecho de dejar de percibir ingresos significó vivir el período de desempleo en pobreza económica. Sin embargo, dicha situación fue más grave en algunos casos que en otros, por lo que fue clasificada en dos grupos: en el primero el desempleo supuso una reducción de los ingresos familiares y en el segundo precariedad económica.

\section{a) Reducción de los ingresos familiares}

En este grupo se encuentran los desempleados cuya aportación económica a la familia es secundaria o parcial (hay otro sostén de la familia). Se trata de amas de casa, hijos de familia, o recién casados con apoyo de los padres. Sólo hubo un cabeza de familia que salió adelante gracias al trabajo de la esposa.

Debido a su condición de sostén secundario, los individuos de este grupo que consiguieron un empleo podían conformarse con un mal sueldo sin que esto afectara significativamente la economía familiar.

Entre quienes optaron por el autoempleo hubo algunos que lo hicieron como una solución temporal al desempleo, "mientras encontraban otra cosa". Otros se dieron cuenta de que el autoempleo era su única opción, ya que difícilmente serían contratados por una empresa. En este último caso se encuentran las amas de casa, los mayores de 40 años o los que carecen de estudios. No obstante que el autoempleo fue una decisión forzosa, y que ganan menos y trabajan más que como empleados, a estas personas el autoempleo les permite cuidar a sus hijos, y/o ser independientes.

\section{b) Precariedad económica}

En este grupo se encuentran los desempleados que no cuentan con la familia como fuente alternativa de ingresos, ya sea porque son ellos la cabeza de familia, o bien porque, aun no teniendo una familia qué mantener, tampoco cuentan con ella para recibir ayuda, generalmente porque son muy pobres. De ahí que la necesidad de salir del desempleo sea más urgente para estos sujetos que para los que poseen otra fuente de ingresos en la familia. Sin embargo, se encuentran diferencias en el grado de precariedad económica, según el nivel socioeconómico del desempleado. 
Para los desempleados de nivel socioeconómico medio o alto la precariedad económica supuso que su nivel de vida pasara de la holgura económica a tener que ajustarse a lo indispensable y prescindir no sólo de gastos superfluos como las salidas al cine o a comer o la compra de juguetes para los hijos, sino también carecer de lo más indispensable. La precariedad económica implicó que no pudieran cubrir las necesidades básicas de la familia (alimentos, servicio de agua, luz). Aun vendiendo sus bienes y llenándose de deudas los hijos de algunos tuvieron que abandonar la escuela y llegaron incluso a pasar hambre.

En cambio, para los de nivel socioeconómico bajo, (que no cuentan con tarjetas de crédito para endeudarse, ni casa o automóvil qué vender) la falta de empleo supuso llegar incluso a la indigencia.

Las vías de superación del desempleo que utilizaron los sujetos en precariedad económica también dependieron de su nivel socioeconómico. Los de posición social media o alta, optaron por el autoempleo. Entre los de nivel socioeconómico bajo no hubo ningún autoempleado. Los sujetos con locus interno de control emigraron para buscar un empleo en Estados Unidos y un sujeto que también vivió la indigencia, pero que tiene locus externo de control, nunca se planteó otra opción más que el encontrar trabajo, aunque fuera temporal.

\section{El desempleo a Nivel Subjetivo}

De acuerdo con los teóricos de la precariedad, subjetivamente el desempleo provocaría inseguridad y vulnerabilidad en los sujetos. En efecto, según los relatos analizados en este estudio, el sentimiento más generalizado entre los desempleados es la inseguridad 0 inestabilidad, la incertidumbre, y el miedo o inseguridad del futuro (12 personas). Entre los jóvenes la inseguridad se expresó como sentir dudas, confusión o inseguridad personal (4 personas).

En cuanto a la vulnerabilidad, aunque ésta no fue manifestada como tal, se mencionó la frustración, la impotencia, el sentimiento de que se cierran todas las puertas, o de que no se ve la salida, o la impaciencia (9 personas); sin embargo, hay otros sentimientos más frecuentes que la vulnerabilidad. La desilusión (decepción, desencanto, desesperanza, pesimismo, desánimo, cansancio), la experimentaron 11 personas, sobre todo los desempleados con locus externo de 
control. También fue más frecuente la desesperación (10 personas), tanto en los empleados como en los autoempleados. Le siguieron la angustia, preocupación, ansiedad o tensión (7 personas).

Algunos sentimientos menos frecuentes, pero comunes a los desempleados con locus externo de control fueron la tristeza o el llanto, y el abatimiento o sufrimiento (5 personas), además de la rabia, el rencor, la envidia de los que tienen seguro social (3 personas). Dichos sentimientos están de acuerdo con el culpar a otros de su situación, propio de las personas con locus externo de control.

En ese sentido, sentimientos comunes a los desempleados con locus interno de control fueron el sentirse desgraciado, miserable, fracasado (3 personas); dichos sentimientos muestran cómo las personas con locus interno de control se culpan a sí mismos de su situación.

Es llamativo que, mayoritariamente quienes se autoemplearon, percibieron que el desempleo provocó en su medio social comentarios críticos tales como: "tanto estudio, ¿para qué?", o ser considerado flojo, atenido, mantenido, vago, bueno para nada. Lo anterior, los hizo sentirse inútiles, "basura", avergonzados, despreciados, rechazados.

A los autoempleados con locus interno de control, el desempleo les causó además crisis existencial y pérdida del sentido de la vida (sentir que no valen, que sería mejor morir), a pesar de contar con el apoyo moral de su familia.

Pero más llamativo aún es que, después de que algunos llegaron a abusar del alcohol o a sufrir depresión, el recurso que más influyó en su decisión de autoemplearse fue el locus interno de control, es decir, confiar en que para autoemplearse no hay que depender más que de sí mismo, del propio esfuerzo y trabajo duro, no de otras personas. Para ello fue fundamental sacar impulso de la adversidad, con la seguridad de que la responsabilidad de crecer es personal, y de que hay que hacer de la crisis una oportunidad.

\section{Perfil emprendedor de los desempleados}

De los 16 autoempleados que participaron en el estudio, 9 cuentan con locus interno de control (56.25\% de los autoempleados) y 7 tienen locus externo (43.75\% de los autoempleados). De los 
14 empleados, 9 tienen locus interno de control (64.3\% de los empleados) y 5 tienen locus externo (35.7\% de los empleados).

\section{a) Género}

Tabla 3

Género. Relatos de desempleados

\begin{tabular}{cll}
\hline Locus interno & Autoempleados 9 & Empleados 9 \\
Femenino & $3-33.3 \%$ & $2-22.2 \%$ \\
Masculino & $6-66.6 \%$ & $7-77.8 \%$ \\
Locus externo & Autoempleados 7 & Empleados 5 \\
Femenino & $2-28.6 \%$ & - \\
Masculino & $5-71.4 \%$ & $5-100 \%$ \\
\hline
\end{tabular}

Fuente: Elaboración propia.

Los autores de los 30 relatos válidos son 23 hombres y 7 mujeres. De los 16 autoempleados 5 son mujeres y 11 son hombres. De los 14 empleados 2 son mujeres y 12 son hombres. Las mujeres se concentran entre las autoempleadas. La mayoría de las mujeres presentaron locus interno de control.

\section{b) Edad}

\section{Tabla 4}

Edad. Relatos de desempleados

\begin{tabular}{cll}
\hline Locus interno & Autoempleados 9 & Empleados 9 \\
20 a 30 años & $1-11.1 \%$ & $3-33.3 \%$ \\
31 a 40 años & $4-44.4 \%$ & $3-33.3 \%$ \\
41 a 50 ó más & $4-44.4 \%$ & $3-33.3 \%$ \\
Locus externo & Autoempleados 7 & Empleados 5 \\
20 a 30 años & $2-28.6 \%$ & $4-80 \%$ \\
31 a 40 años & $2-28.6 \%$ & \\
41 a 50 ó más & $3-42.8 \%$ & $1-20 \%$ \\
\hline
\end{tabular}

Fuente: Elaboración propia.

Los individuos con locus interno de control son los de mayor edad y los jóvenes se concentran entre quienes tienen locus externo de control. 


\section{c) Estado civil}

\section{Tabla 5}

Estado civil. Relatos de desempleados

\begin{tabular}{cll}
\hline Locus interno & \multicolumn{1}{c}{ Autoempleados 9} & Empleados 9 \\
Soltero & $2-22.2 \%$ & $4-44.4 \%$ \\
Casado & $6-66.6 \%$ & $5-55.5 \%$ \\
Divorciado & $1-11.1 \%$ & - \\
Locus externo & Autoempleados 7 & Empleados 5 \\
Soltero & $2-28.6 \%$ & $3-60 \%$ \\
Casado & $3-42.8 \%$ & $2-40 \%$ \\
Divorciado & $2-28.6 \%$ & - \\
\hline
\end{tabular}

Fuente: Elaboración propia.

En este estudio, la mayoría de los autoempleados eran casados y los empleados solteros. Esto es hasta cierto punto de esperarse, dado que los autoempleados son menos jóvenes que los empleados.

\section{d) Nivel socioeconómico}

Tabla 6

Nivel socioeconómico. Relatos de desempleados

\begin{tabular}{cll}
\hline Locus interno & Autoempleados 9 & Empleados 9 \\
Alto & $1-11.1 \%$ & - \\
Medio & $3-33.3 \%$ & $6-66.7 \%$ \\
Bajo & $5-55.6 \%$ & $3-33.3 \%$ \\
Locus externo & Autoempleados 7 & Empleados 5 \\
Alto & $2-28.6 \%$ & - \\
Medio & $3-42.8 \%$ & $2-40 \%$ \\
Bajo & $2-28.6 \%$ & $3-60 \%$ \\
\hline
\end{tabular}

Fuente: Elaboración propia.

Según el nivel socioeconómico declarado por los sujetos de este estudio, los únicos individuos de clase alta son autoempleados. Pero, al mismo tiempo, los porcentajes más altos de 
autoempleados poseen nivel bajo. Los empleados se concentran en el nivel socioeconómico medio.

\section{e) Nivel de escolaridad}

\section{Tabla 7}

Nivel de escolaridad. Relatos de desempleados

\begin{tabular}{cll}
\hline Locus interno & Autoempleados 9 & Empleados 9 \\
Postgrado & $1-11.1 \%$ & $1-11.1 \%$ \\
Profesional & $4-44.4 \%$ & $5-55.6 \%$ \\
Preparatoria & $1-11.1 \%$ & - \\
Secundaria & $2-22.2 \%$ & $2-22.2 \%$ \\
Primaria & $1-11.1 \%$ & $1-11.1 \%$ \\
Locus externo & Autoempleados 7 & Empleados 5 \\
Postgrado & - & - \\
Profesional & $3-42.8 \%$ & $2-40 \%$ \\
Preparatoria & $2-28.6 \%$ & $2-40 \%$ \\
Secundaria & $1-14.3 \%$ & $1-20 \%$ \\
Primaria & $1-14.3 \%$ & - \\
\hline
\end{tabular}

Fuente: Elaboración propia.

Los profesionales se concentran entre quienes tienen locus interno de control, tanto empleados como autoempleados. Esto podría indicar que el contar con educación superior brinda a los individuos confianza en las propias capacidades. 


\section{f) Capital social}

Tabla 8

Capital social. Relatos de desempleados

\begin{tabular}{cll}
\hline Locus interno & Autoempleados 9 & Empleados 9 \\
Familia & $3-33.3 \%$ & $3-33.3 \%$ \\
Amigos & $2-22.2 \%$ & $1-11.1 \%$ \\
Solos & $4-44.4 \%$ & $5-55.6 \%$ \\
Locus externo & Autoempleados 7 & Empleados 5 \\
Familia & $1-14.3 \%$ & $1-20 \%$ \\
Amigos & $5-71.4 \%$ & - \\
Solos & $1-14.3 \%$ & $4-80 \%$ \\
\hline
\end{tabular}

Fuente: Elaboración propia.

Recurrir a la ayuda de la familia o de amigos, es más común entre quien se propone autoemplearse que entre quien busca un empleo. Por otro lado, la tendencia a "salir adelante" solo, sin ayuda, se presenta con frecuencia tanto entre los desempleados con locus interno como entre los que tienen locus externo de control. En los primeros, la confianza que tienen en sí mismos los hace ser más independientes. En los sujetos con locus externo de control, en cambio, es la desconfianza en la ayuda de otros o el pesimismo lo que los lleva a no buscar ayuda. Sin embargo, el motivo es distinto: algunos no buscan ayuda y otros no cuentan con ella. Los desempleados de niveles socioeconómicos más altos, a diferencia de los pobres, cuentan más con la familia como estrategia de sobrevivencia.

\section{DISCUSIÓN}

De acuerdo con los resultados de este estudio, desde el punto de vista económico, los desempleados más precarios serían: Los jefes de familia sin otra fuente de ingresos en la familia, de nivel socioeconómico bajo, mayores de 40 años, o sin estudios. La única alternativa que vislumbran es buscar empleo en Estados Unidos. En segundo lugar, estarían los jefes de familia, sin otra fuente de ingresos, mayores de 40 años. Su vía de superación del desempleo es el autoempleo. Quienes además de estas características cuentan con nivel socioeconómico medio o alto y con educación, suelen apoyarse en redes sociales para salir adelante. 
En tercer lugar, los menos precarios serían aquellos desempleados que no son cabeza de familia. Su opción puede ser un empleo, aunque sea de mala calidad, o si son madres de familia, mayores de 40 años o sin estudios, el autoempleo.

Desde el punto de vista subjetivo, aunque todos son igualmente precarios, los desempleados que no son el principal responsable del sostenimiento de la familia sufren menos vulnerabilidad, ya que su situación económica no es tan crítica.

El resultado más significativo con respecto al perfil precario de los desempleados es que quienes se autoemplearon son los que expresaron haber sufrido más durante el desempleo. Parece que el impulso hacia el autoempleo fue el rechazo social y los sentimientos de falta de sentido de la vida. Lo anterior se expresó como "sacar fuerza de la adversidad", en algunos casos solos, en otros con ayuda de la familia, pero en ningún caso como beneficiarios de algún tipo de apoyo del gobierno.

Con respecto al perfil emprendedor, las diferencias encontradas entre los desempleados que consiguieron un empleo y quienes se autoemplearon son las siguientes:

En cuanto al género, que las mujeres se concentran entre los autoempleados. En numerosos países, incluido México, se ha demostrado que las mujeres trabajan menos horas que los hombres (Marlow \& MacAdam, 2013), u optan por el autoempleo con la idea de hacer compatible esta actividad con las tareas domésticas (Baycant-Levent et al., 2003, p. 14; Kelley et al., 2013). Más aún, la participación de las mujeres en el autoempleo está correlacionada con actitudes culturales y políticas que brindan apoyos a la maternidad (Parker, 2009; Tonoyan, Budig \& Strohmeyer, 2010; Arceo, 2011).

Algunas diferencias de género en el autoempleo provienen del capital humano, la actitud ante el riesgo y las distintas motivaciones que tienen los hombres y las mujeres. Al respecto, aunque las mujeres posean más años de escolaridad, suelen tener menos conocimientos financieros y ser más adversas al riesgo. Como consecuencia, las mujeres tienden a operar negocios más pequeños, a invertir menos en ellos y, por lo tanto, a obtener menos ganancias (Parker, 2009; Baycant \& Nijkamp, 2001). 
En cuanto a las motivaciones para iniciar un negocio, las mujeres se muestran tan motivadas por el logro y la independencia como los hombres; sin embargo, parece que la independencia en el lugar de trabajo ("ser su propio jefe"), junto con los incentivos económicos (hacer más dinero), son más importantes para los hombres que para las mujeres, para quienes combinar familia y trabajo es más valorado (Parker, 2009; Brush et al., 2004).

Con respecto a la edad, los resultados de este estudio coinciden con los encontrados por otros autores cuando señalan que los autoempleados tienden a ser menos jóvenes que los empleados. En México, se sabe que en el caso concreto de los desempleados entre 18 y 25 años de edad, existe una mayor probabilidad de que sean empleados formales que autoempleados (CalderónMadrid 2008, cit., en Arceo, 2011, p. 45).

Las diferencias con respecto al nivel socioeconómico, consisten en que los empleados se ubican en el nivel medio y los autoempleados en los extremos alto y bajo. Al respecto, los estudios sobre movilidad social discuten si las habilidades, la ambición y el esfuerzo personales aportan más que la clase social de origen para ascender socialmente. En Inglaterra, se ha encontrado que el origen social alto influye positivamente en el éxito del negocio emprendido (Blackburn et al., 2013; Golthorpe \& Mills 2008; Goldthorpe and Jackson, 2007). Lo anterior, parece también ser cierto en América Latina. Al respecto, Álvarez (2013) presenta evidencias que sugieren que los emprendedores Latinoamericanos "cuyos padres eran "más ricos" al momento de cumplir la mayoría de edad son más propensos a generar emprendimientos de mayor tamaño" (Álvarez, 2013, p. 189).

En lo referente al capital social, el presente estudio mostró que recurrir a la ayuda de la familia 0 de amigos es más común entre quien se propone autoemplearse que entre quien busca un empleo. El capital social es importante porque es el medio de obtener información que facilite el acceso a recursos y clientes, al mismo tiempo que provee apoyo psicológico al autoempleado (Parker, 2009). Existen evidencias de que en México las redes sociales son más efectivas para encontrar un empleo informal o autoemplearse y que para el empleo formal se utiliza más el contacto directo (Arceo, 2011).

Las características similares entre los empleados y los autoempleados del estudio que aquí se presentan, son las que se refieren al estado civil: la mayoría son casados. En este sentido, Salas 
(2003), señala que, si los desempleados tienen una familia qué mantener y no cuentan con ahorros $u$ otra fuente de ingreso familiar, tienen que hacer "una búsqueda menos restrictiva, lo que facilita la inserción en un puesto de trabajo (incluidas las actividades por cuenta propia)" (Salas, 2003:138). De ahí que -según este autor- en México, más del 50\% de los desocupados consiga empleo en el lapso de un trimestre, ya que no se pueden dar el lujo de permanecer desempleados (Ibid, pp. 137-139). En el caso de las mujeres, si son casadas, tienen más probabilidades que los hombres de que su salida del desempleo sea hacia el autoempleo (Arceo, 2011, p. 47).

Con respecto al nivel de escolaridad la literatura señala que la educación formal tiene un significativo y positivo efecto en la tendencia a convertirse en autoempleado en algunos países, pero más ambiguos efectos en otros. Por ejemplo, en Estados Unidos se ha encontrado correlación positiva entre mayor educación y autoempleo, pero en Europa y en países en desarrollo esta correlación es negativa (Baycan-Levent et al., 2006; Van der Sluis et al., 2005). En Colombia y México, por ejemplo, los individuos con más años de escolaridad tienen una mayor probabilidad de ser empleados que de ser autoempleados (Mejía and Meléndez, 2012; Negrete, 2001). En México, los más educados ingresan al empleo o al autoempleo en el sector formal (Arceo, 2011), mientras que los menos educados (y generalmente de bajo nivel socioeconómico) tienen mayor tendencia a insertarse en el sector informal, ya sea como empleados o como autoempleados (Rodríguez-Oreggia, 2007). Esto es así, porque quienes han invertido más en su educación son más selectivos, no aceptan cualquier ocupación sin haber dedicado tiempo en la búsqueda (Negrete, 2001). Pero, como se mostró al hablar de nivel socioeconómico, esto sólo es posible para quien cuenta con recursos económicos suficientes para sobrevivir varios meses sin ingresos. Generalmente éstos provienen de la familia u otras redes de apoyo.

No obstante, lo anterior, en este estudio se encuentra que los profesionales se emplean o autoemplean indistintamente. En cuanto al locus de control diversos estudios indican que los individuos con locus interno de control tienden más a iniciar un negocio que los individuos con locus externo de control (Mueller y Thomas, 2000; Frantz, 1980). Sin embargo, esta relación varía de acuerdo al país. Gurel, Altinay y Danielle (2010), reportan que entre estudiantes de Turquía y de Gran Bretaña no existe una influencia significativa del locus interno de control en la intención emprendedora. Por su parte, Ang y Hong (2000), sí encuentran tal influencia en Singapur y en menor medida en Hong Kong, lo que dichos autores atribuyen a diferencias culturales. En México 
se ha encontrado que la actitud emprendedora es más frecuente entre los estudiantes universitarios que poseen locus interno de control (Correa y Aduna, 2012; Espíritu, 2011).

El resultado más llamativo de esta investigación es que no se encontró una marcada diferencia de locus de control entre empleados y autoempleados: en ambos grupos existen individuos que cuentan con locus interno de control. Esto hace pensar que el desempleo requiere de talento y confianza en las propias capacidades para superarlo, ya sea por la vía del empleo o por la del autoempleo.

Teniendo en cuenta lo anterior se puede afirmar que el autoempleo parece más una opción precaria que emprendedora. Sin embargo, también se puede sostener que aun en la situación más precaria hay desempleados que muestran su capacidad de emprender.

\section{CONCLUSIONES}

Las bajas tasas de desocupación en México muestran que el mercado de trabajo se basa en un dinamismo de sobrevivencia y en la tendencia a delegar en los individuos la creación de sus propios medios de trabajo, sin apoyos y en la más grave vulnerabilidad. De ahí que, aunque los sujetos tengan la capacidad de generar sus propios empleos, éstos no puedan ser de calidad, dada la precariedad en la que se encuentran quienes se han visto en la necesidad de autoemplearse. En este sentido se podría decir que los sectores informales han actuado como receptáculo de la mano de obra que no encuentra acomodo y, desalentada, se integra a formas poco productivas o precarias de trabajo, como el autoempleo. En principio, dichas formas resuelven la supervivencia y en algunos casos logran madurar como empleos de inversión.

A este respecto, de acuerdo con el GEM (Acs, 2006), se debería evitar el emprendedurismo por necesidad (no orillar a los sujetos a tal situación), por medio de la creación de más empleos formales. Por otro lado, los apoyos a la creación de micronegocios deberían estar dirigidos a los emprendedores por oportunidad.

Lo anterior se afirma partiendo de que los autoempleados no son verdaderos emprendedores. Sin embargo, en este estudio se ha encontrado que los autoempleados que provienen del desempleo son personas con el rasgo emprendedor del locus interno de control, pero que han sido precarizados. Esto quiere decir que, si se atendiera a las recomendaciones del GEM, los 
autoempleados no serían candidatos a apoyos para emprendedores, cuando en realidad son personas que aun en el estado de gran vulnerabilidad en que se encuentran demuestran que pueden ser muy productivos.

Para concluir se puede decir que en cuestión de empleo existe un problema que no ha sido atendido: la falta de apoyo a los autoempleados de acuerdo a su perfil y sus capacidades. En este artículo se ha mostrado que un desempleado que es ama de casa, tiene más de 40 años o carece de estudios suficientes, si no se autoemplea, puede llegar al nivel más extremo de la pobreza, pues no cuenta con otras opciones de empleo.

En síntesis: el autoempleo se revela más como una opción precaria que emprendedora. Más aún, parece que el impulso hacia el autoempleo es la precariedad. Sin embargo, también se puede sostener que los desempleados más precarios superan el desempleo gracias al rasgo emprendedor del locus interno de control. Por ello resulta relevante reconocer no sólo el perfil precario, sino también el perfil emprendedor de quienes se han visto forzados a autoemplearse, para apoyarlos de acuerdo con sus necesidades específicas y su talento empresarial. 


\section{REFERENCIAS BIBLIOGRÁFICAS}

Acs, Z. (2006). How Is Entrepreneurship Good for Economic Growth? Innovations: technology, governance, globalization, 1(1), 97-107.

Álvarez, F. (2013). Transformación productiva y empresas de calidad. En: Sanguinetti, P., Brassiolo, P., Arreaza, A., Berniell, L., Álvarez, F., Ortega, D. \& Kamiya, M. Emprendimientos en América Latina. Desde la subsistencia hacia la transformación productiva (pp. 155-202). Bogotá: Corporación Andina de Fomento (CAF).

Amin, A. y Robins, K. (1991). Distritos industriales y desarrollo regional: límites y posibilidades. Sociología del Trabajo, número extraordinario, 131-229.

Ang, S. H. \& Don H. (2001). Entrepreneurial spirit among East Asian Chinese. Thunderbird International Business Review, 42(3), 285-309.

Arceo Gómez, E. (2011). Estudio cuantitativo sobre desempleo en México y sus implicaciones para la participación laboral femenina. México D.F.: Instituto Nacional de las Mujeres.

Arum, R. \& Müller W. (2009). The Reemergence of Self-Employment: A Comparative Study of Self-Employment Dynamics and Social Inequality. New York: Princeton University Press.

Baycan-Levent, T., Masurel, E. \& Nijkamp, P. (2006). Gender differences in ethnic entrepreneurship. International Journal of Entrepreneurship and Innovation Management, 6(3), 173-190.

Baycan-Levent, T. \& Nijkamp P. (2011). Migrant Female Entrepreneurship: Driving Forces, Motivation and Performance. En: Desai, S., Nijkamp, P. \& Stough, R. (Ed.) New Directions in Regional Economic Development: The Role of Entrepreneurship Theory and Methods, Practice and Policy (pp. 198-236). Cheltenham and Northampton: Edward Elgar Publishing.

Beck, U. (2007). Un nuevo mundo feliz. La precariedad del trabajo en la era de la globalización. Madrid: Paidós.

Blackburn, R., Conelly, R., Lambert, P. \& Gayle, V. (2013). Social stratification: trends and processes. United Kingdom: Ashgate Publishing.

Blanchflower, D. (2004). Self-Employment: more may not be better. Swedish Economic Policy Review, 11(2), 15-74.

Boltanski, L. \& Chiapello, E. (2002). El nuevo espíritu del capitalismo. Madrid: Akal. 
PERFIL EMPRENDEDOR Y PRECARIO DE DESEMPLEADOS QUE SE AUTOEMPLEAN

Brassiolo, P. y Arreaza, A. (2013). ¿Quiénes son los emprendedores en América Latina? En: Sanguinetti, P., Brassiolo, P., Arreaza, A., Berniell, L., Álvarez, F., Ortega, D. \& Kamiya, M. Emprendimientos en América Latina. Desde la subsistencia hacia la transformación productiva (pp. 65-111). Bogotá: Corporación Andina de Fomento (CAF).

Caliendo, M., Fossen, F. \& Kritikos, A. (2014). Personality Characteristics and the Decision to Become and Stay Self-Employed. Small Business Economics, 42(4), 787-814.

Castel, R. (1997). La metamorfosis de la cuestión social. Buenos Aires: Paidós.

Correa López, M.I. y Aduna Mondragón. A.P. (2012). Actitud emprendedora en estudiantes de la Licenciatura en Administración, mediante el uso de modelación de ecuaciones estructurales. Denarius, 25(1), 75-95.

Davidsson, P. \& Honig, B. (2003). The role of social and human capital among nascent entrepreneurs. Journal of Business Venturing, 18(3), 301-331.

De la Garza, E. (2001). La Formación Socioeconómica Neoliberal: debates teóricos acerca de la reestructuración de la producción y evidencia empírica para América Latina. México, D.F.: Plaza y Valdés.

De Oliveira, O. y García, B. (1998). Crisis, reestructuración económica y mercados de trabajo en México. Papeles de Población, 4(15), 39-72.

De Oliveira, O. (2006). Jóvenes y precariedad laboral en México. Papeles de Población, 12(49), 37-73.

Douglas, E. \& Shepherd, D. (2002). Self-employment as a Career Choice: Attitudes, Entrepreneurial Intentions, and Utility Maximization. Entrepreneurial Theory and Practice, 26(3), 81-90.

Dunn, T. \& Douglas, H.E. (2000). Financial Capital, Human Capital and the Transition to SelfEmployment: Evidence from Intergenerational Links. Journal of Labor Economics, 18(2), 282-305.

Espíritu Olmos, R. (2011). Análisis de la intención emprendedora en estudiantes universitarios a través de los rasgos de personalidad. Multiciencias, 11(1), 65-75.

Frantz, R. (1980). Internal-external locus of control and labor market performance. Empirical evidence using longitudinal survey data. A Quarterly Journal of Human Behavior, 17(3), 2329. 
Goldthorpe, J. \& Jackson, M. (2007). Intergenerational class mobility in contemporary Britain: political concerns and empirical findings. British Journal of Sociology, 58(4), 525-546.

Goldthorpe, J. \& Mills, C. (2008). Trends in intergenerational class mobility in modern Britain: evidence from national surveys, 1972-2005. National Institute Economic Review, 205(1), 83-100.

Gurel, E., Altinay, L. \& Daniele, R. (2010). Tourism Students' Entrepreneurial Intentions. Annals of Tourism Research, 37(3), 646-669.

Kalleberg, A. (2009). Precarious work, insecure workers: employment relations in transition. American Sociological Review, 74(1), 1-22.

Kelley, D., Brush, C., Greene, P. \& Litovsky, Y. (2013). Global Entrepreneurship Monitor: 2012 Women's Report. Boston: The Center for Women's Leadership at Babson College and London Business School.

Lederman, D., Messina, J., Pienknagura, S. \& Rigolini, J. (2014). Latin American entrepreneurs. Many firms but little innovation. Washington D.C.: World Bank.

Marlow, S. \& McAdam, M. (2013). Gender and Entrepreneurship: Advancing debate and challenging myths; exploring the mystery of the under-performing female entrepreneur. International Journal of Entrepreneurial Behavior and Research, 19(1), 114-124.

Mueller, S. \& Thomas, A. (2000). Culture and entrepreneurial potential: a nine country study of locus of control and innovativeness. Journal of Business Venturing, 16(1), 51-75.

Parker, S. (2009). The Economics of Entrepreneurship. United Kingdom: Cambridge University Press.

Rodgers, G. \& Rodgers, J. (1989). Precarious jobs in labour market regulation: the growth of atypical employment in Western Europe. Geneva: International Institute for Labour Studies.

Rodríguez-Oreggia, E. (2007). La dinámica comparativa del sector informal en México. Documentos de Investigación. México D.F.: Instituto de Investigaciones sobre Desarrollo Sustentable y Equidad Social, Universidad Iberoamericana.

Rotter, J. (1990). Internal versus external control of reinforcement. A case history of a variable. American Psychologist, 45(4), 489-493. 
PERFIL EMPRENDEDOR Y PRECARIO DE DESEMPLEADOS QUE SE AUTOEMPLEAN

Salas, C. (2003). Trayectorias laborales entre el empleo el desempleo y las microunidades en México. Papeles de Población, 9(38), 121-157.

Sanguinetti, P. (2013). El emprendimiento como motor del desarrollo, En: Sanguinetti, P., Brassiolo, P., Arreaza, A., Berniell, L., Álvarez, F., Ortega, D. \& Kamiya, M. Emprendimientos en América Latina. Desde la subsistencia hacia la transformación productiva (pp.19-61). Bogotá: Corporación Andina de Fomento (CAF).

Sennett, R. (2007). Relatos del tiempo de la precariedad. Nexos, 1(349), 21-26.

Stephan, U., Hart, M. \& Drews, C.C. (2015). Understanding motivations for entrepreneurship. A review of recent research evidence. Birmingham: Enterprise Research Centre.

Storey D. \& Johnson, S. (1987). Job Generation and Labour Market Change. Basingstoke: MacMillan.

Tonoyan, V., Budig, M. \& Strohmeyer, R. (2010). Exploring the heterogeneity of women's entrepreneurship: the impact of family structure and family policies in Europe and the US. En: Brush, C., De Bruin, A., Gatewood, E. \& Henry, C. (Ed.) Women Entrepreneurs and the Global Environment for Growth: A Research Perspective (pp.137-162). Cheltenham and Northampton: Edward Elgar Publishing.

Van der Sluis, J., Van Praag, M. \& Vijverberg, W. (2005). Entrepreneurship Selection and Performance: A meta-analysis of the impact of education in developing economies. The World Bank Economical Review, 19(2), 225-261.

White, M.D. \& Marsh, E.E. (2006). Content analysis: a flexible methodology. Library trends, 55(1), 22-45.

\section{Fuentes electrónicas:}

Audretsch D.B., Carree M.A. \& Thurik A.R. (2001). Does Entrepreneurship Reduce Unemployment? Discussion Paper 01-074/3, Amsterdam and Rotterdam: Tinbergen Institute. Recuperado de: http://hdl.handle.net/10419/85927. Consultado: 26/07/2015.

Baycan-Levent, T., Ahu Gülümser, A., Kundak, S., Nijkamp, P. \& Sahin, M. (2006). Diversity and ethnic entrepreneurship. Position Paper of Research, Task 4. Recuperado de: http://www.susdiv.org/uploadfiles/RT4_4_PP_Tuzin.pdf. Consultado: 23/11/2015.

Brush, C., Carter, N., Gatewood, E., Greene, P. \& Hart, M. (2004). Women entrepreneurs, Growth and implications for the classroom. Coleman Foundation White Paper Series for the United 
States Association for Small Business and Entrepreneurship: Entrepreneurship in a Diverse World, 47-91. Recuperado de:

http://citeseerx.ist.psu.edu/viewdoc/download?doi=10.1.1.210.3001\&rep=rep1\&type=pdf. Consultado: 4/12/2015.

De la Garza, E. y Gaspar, H. (2010). Modelos de producción y mercado de trabajo de profesionistas en México. Working Papers 04. California: Institute for Research on Labor and Employment. Recuperado de: http://eprints.cdlib.org/uc/item/87p115d6. Consultado: 26/04/2016.

Frade, C., Darmon, I. \& Laparra, M. (2004). Precarious employment in Europe: a comparative study of Labour market related risks in flexible economies. Final report, ESOPE Project. Brussels: European Comission. Recuperado de:

ftp://ftp.cordis.europa.eu/pub/citizens/docs/kina21250ens_final_esope.pdf.

Consultado: 19/02/2016.

International Labour Organization (ILO). (2012). From precarious work to decent work. Outcome document to the workers' symposium on policies and regulations to combat precarious employment. Geneva: International Labor Office. Recuperado de: http://www.ilo.org/wcmsp5/groups/public/---ed_dialogue/--actrav/documents/meetingdocument/wcms_179787.pdf. Consultado: 26/04/2016.

Instituto Nacional de Estadística y Geografía. (2012). Encuesta nacional de ocupación y empleo. Recuperado de:

http://www.inegi.org.mx/sistemas/olap/proyectos/bd/consulta.asp?p=17606\&c=10819\&s=est\&cl =4. Consultado: 19/01/2012.

Instituto Nacional de Estadística y Geografía. (2010). Indicadores oportunos de Ocupación y Empleo. Comunicado No. 432/10. Recuperado de:

http://www.canaintex.radius.com.mx/envios/doctos/247_inegi.pdf. Consultado: 22/12/2010.

Mejía, P. \& Meléndez, M. (2012). Middle-Class Entrepreneurs and Social Mobility through Entrepreneurship in Colombia. Inter-American Development Bank Working Paper Series No. $317 . \quad$ Recuperado de: http://idbdocs.iadb.org/wsdocs/getdocument.aspx?docnum=37123490. Consultado: 26/04/2016. 
Negrete Prieto, R. (2001). ¿Por qué han sido bajas las tasas de desempleo abierto en México?: una guía básica ilustrada. Notas, Revista de información y análisis, (15), 7-13. Recuperado de:

http://www.inegi.org.mx/prod_serv/contenidos/espanol/bvinegi/productos/integracion/espe ciales/notas/notas15.pdf. Consultado: 12/10/2014.

OECD. (2014). Society at a Glance 2014: OECD Social Indicators. OECD Publishing. Recuperado de: http://dx.doi.org/10.1787/soc_glance-2014-en. Consultado: 10/10/2014.

OCDE. (2013). Temas y políticas clave sobre PYMEs y emprendimiento en México. OECD Publishing. Recuperado de: http://dx.doi.org/10.1787/9789264204591-es. Consultado: $12 / 10 / 2014$.

Organización Internacional del Trabajo. (2013). Panorama Laboral 2013, América Latina y el Caribe. Recuperado de: http://www.ilo.org/americas/publicaciones/panoramalaboral/WCMS_232760/lang--es/index.htm. Consultado: 10/10/2014.

Secretaría del Trabajo y Previsión Social. (2014). Información Laboral 2014. Recuperado de: http://www.stps.gob.mx/bp/secciones/conoce/areas_atencion/areas_atencion/web/pdf/perf iles/perfil\%20nacional.pdf. Consultado: 10/10/2014. 